\title{
Particle interactions in oscillatory Stokes flow
}

\author{
M. S. Ingber ${ }^{1} \&$ P. Vorobieff ${ }^{2}$ \\ ${ }^{1}$ Department of Mechanical Engineering, University of Colorado Denver, \\ Denver, CO 80217, USA \\ ${ }^{2}$ Department of Mechanical Engineering, University of New Mexico, \\ Albuquerque, NM 87131, USA
}

\begin{abstract}
It has been observed both experimentally and numerically for relatively dense suspension flows that small-amplitude oscillating Poiseuille flow results in particle migration towards the high shear rate region of the flow field (pipe walls), whereas, large-amplitude oscillating Poiseuille flow results in particle migration towards the low shear rate region of the flow field (pipe centerline). To date, there has been no satisfactory explanation as to why the period of oscillation has this effect on the direction of particle migration. In the current paper, we demonstrate through numerical simulation the same behavior in two-particle systems which provides a first principle explanation of at least one cause for this phenomenon in dense suspension flows. The numerical analysis is based on a semi-analytic solution for the motion of two spheres suspended in arbitrary, unbounded shear flow.
\end{abstract}

\section{Introduction}

Particle motion in nonlinear shear flows is playing an important role in a variety of developing technologies including the production of semiconductors and magnetic recording media, processing of energetic materials, encapsulation of electronic components, chromatography, secondary oil recovery by hydraulic fracturing, carbon-dioxide sequestration, and transport of contaminants in semiconductor and photomask technologies, to name a few. A common outstanding fundamental research issue associated with these technologies is the development of the relationship between microstructural interactions and macroscopic system behavior. 
Experiments performed over the past 30 years have established that particles suspended in nonlinear shear flows such as Poiseuille and Couette flows migrate from regions of high shear rate and particle concentration to regions of low shear rate and particle concentration [1,2]. However, the underlying physics has not been completely elucidated, resulting in less than satisfactory rheological models. In particular, current rheological models have been deficient in several regards including the scaling of particle migration rate with respect to characteristic particle size, incorporation of slip boundary conditions, and nonisotroic effects caused by particle agglomeration and chaining [3,4].

Another shortcoming of current rheological models is that they all predict that the particle migration direction will not change if the flow direction is reversed. For large period oscillatory flows, although there will be some problems in the short term transient as particle chains flip orientation, this is not a huge concern. However, for small period pressure-driven oscillatory flows, it has been observed, both through experiment [5] and numerically [6], that the direction of particle migration actually switches towards the high shear rate region of the flow field. That is, for example, in oscillating Poiseuille flow in a pipe, particles will migrate towards the axis of the pipe (low shear region) if the period of the oscillation is above a critical value and will migrate towards the pipe wall (high shear region) if the period is below a critical value. The investigators who made this stunning observation experimentally and numerically recognized that no current rheological model captures this phenomenon. However, they did not put forward a theory to explain it.

In the current research, we perform high-fidelity numerical simulations of a pair of identical spheres in oscillating nonlinear shear flow. We are able to determine, for a specified set of initial conditions a critical period above which the particle pair will migrate towards the low shear rate region of the flow field and below which the particle pair will migrate towards the high shear rate region of the flow field. By analyzing the particle pair interactions, we put forward a theory for the change of direction in suspension flows.

\section{Numerical methodology}

We presume in this research that the Reynolds number is small enough so that both particle and fluid inertia can be ignored. Hence, the governing equation for the fluid is the Stokes equation and the governing equations for the particles are equilibrium and kinematic equations. The numerical method used to perform the simulations in the current research is based on a semi-analytic solution for the motion of two spheres suspended in an unbounded but otherwise arbitrary shear flow [7]. The semi-analytic method is a vast generalization of classical bisphericalcoordinate solutions for two spheres moving along or perpendicular to their line of centers, rotating about the centerline in a quiescent liquid, or suspended in linear shear flow. The method is highly efficient and very convenient since the algorithm does not require any local expansions of the ambient velocity field $u_{\infty}(x)$, but simply operates with the values of $u_{\infty}(x)$ in the vicinity of the spheres, which 
can be calculated either by an analytic formula (e.g., for Poiseuille flow) or by a user-provided routine for more complex cases. The method provides linear and angular velocities as a function of position. Once the linear and angular velocities are determined, the particles are repositioned in space using a third-order, variabletime-step Runge-Kutta routine.

The governing equations considered in this research are kinematically reversible, which, among other things, means that for purely Stokes flow driven by periodic forcing or periodic boundary motion (e.g., Couette flow produced by translation of a plate that then reverses direction and returns to its original position), the net motion of any fluid particle over one period is zero.

Further, this particular numerical algorithm is extremely accurate, and hence, the particle motions for presumed smooth spheres are essentially reversible. We have shown previously that, in order to experience particle migration as observed in experiment, both surface roughness and a nonlinear shear field are required [8]. Since we do want to study particle migration, we add a roughness model. The roughness model considered in this research is essentially equivalent to the nonlocked model of DaCunha and Hinch [9] in which particles are not allowed to have separations less than a specified roughness $\varepsilon$, but are allowed to rotate relative to each other.

\section{Results}

We first consider the case of the interaction of two spheres of equal radius in non-oscillating, nonlinear shear flow. The following definitions are useful in characterizing these problems. The coordinates of the two particles are given by $\left(x_{1}, y_{1}, z_{1}\right)$ and $\left(x_{2}, y_{2}, z_{2}\right)$. The initial center to center separations of the spheres in the three coordinate directions are given by $\Delta x_{-\infty}, \Delta y_{-\infty}$, and $\Delta z_{-\infty}$. The shear plane is the $x-z$ plane, the far-field velocity is in the x-direction, and the initial separation is given by $\Delta x_{-\infty}=-10 a$, where $a$ is the sphere radius. The quadratic ambient flow field is directed along the $x$-axis and is given by $u(z)=1.08 \overline{3}-0.75 *(z-0.6 \overline{6})^{2}$. The particles have initial $x, y$, and $z$ coordinates given by $(-5.0,0.0,0.05)$ and $(5.0,0.0,-0.05)$. Upon approach (as shear brings the particles closer together) the $z$-coordinate of the center of mass of the particle pair, $z_{c m}$, moves towards the low shear rate region of the flow field which is in the positive $z$-direction. Upon separation (as the particle pair is split by shear), the center of mass of the particle pair moves back towards the high shear rate region of the flow field. Any particle roughness destroys the symmetry in the particle pair trajectory, causing a net migration towards the low shear rate region. This is shown in Fig. 1 where the vertical location of the center of mass of the particle pair is plotted against the center-to-center separation in the $x$-direction, $\Delta x$. As seen in the figure, the $z$-coordinate of the center of mass, $z_{\mathrm{cm}}$, increases upon approach $(\Delta x<0)$. That is, upon approach the particle pair migrates towards the low shear rate region of the flow field. Upon separation $(\Delta x>0)$, the particle pair migrates back towards the high shear rate region of the flow field. For smooth particles $(\varepsilon=0)$, there is symmetry about $\Delta x=0$ so that the migration towards 
the low shear rate region upon approach is of the same total magnitude as the migration back towards the high shear rate region upon separation resulting in no net migration. The combination of particle roughness and the nonlinear shear results in a net particle migration towards the low shear rate region of the flow field as seen in the figure. It is also observed in the figure that the net particle migration increases with increasing particle roughness.

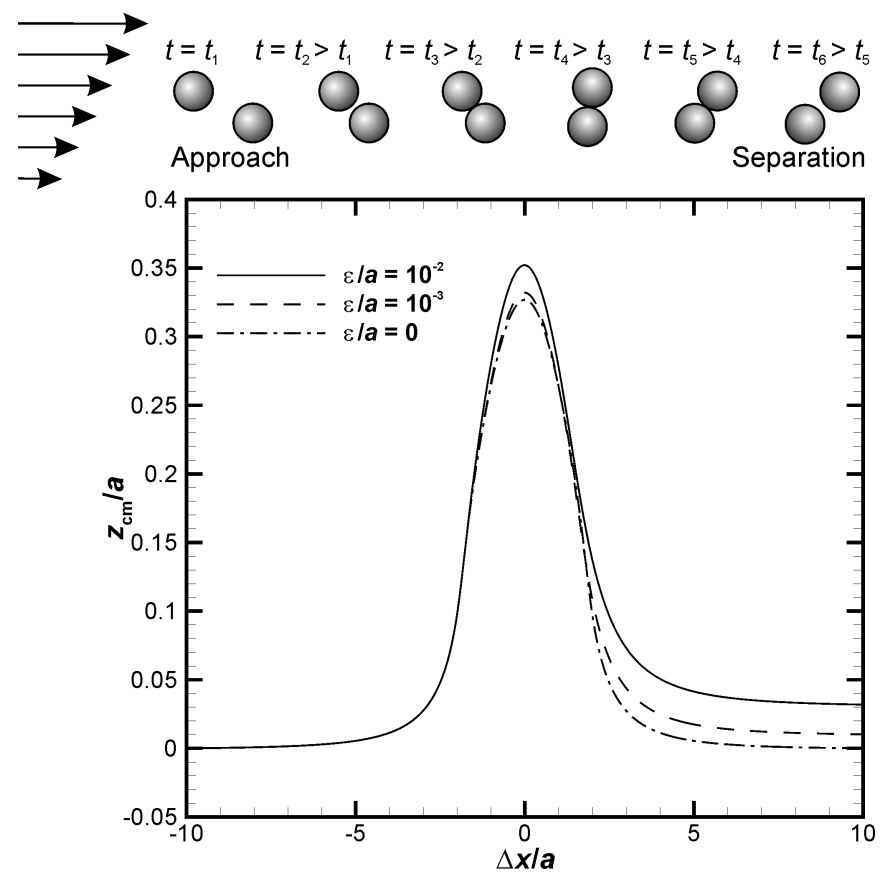

Figure 1: Top: schematic of the interaction of two spheres in shear flow, showing approach, contact, and separation. Bottom: the vertical location of the center of mass, $z_{c m} / a$, as a function of $\Delta x / a$ for two spheres suspended in nonlinear shear flow with initial positions $(-5.0,0.0,0.05)$ and $(5.0,0.0,-$ 0.05 ). Plots for three particle roughnesses (relative roughness $\varepsilon / a=0$, $10^{-3}$, and $10^{-2}$ ) are shown.

The reason for the net particle migration towards the low shear rate region of the flow field is somewhat subtle. Particle roughness causes an asymmetry in the particle trajectories: at a given angle between the line-of-center between the particle pair and the ambient flow direction, roughness drives the spheres slightly farther apart on separation compared to approach. However, at a specified angle, the migration velocity actually increases with increasing separation. As an example, Fig. 2 shows the absolute value of the average velocity of the particle pair in the $z$-direction, $\left|w_{c m}\right|$, as a function of the surface-to-surface separation, $\delta / a$, for the case in which the line of center of the particle pair makes an angle of $45^{\circ}$ 


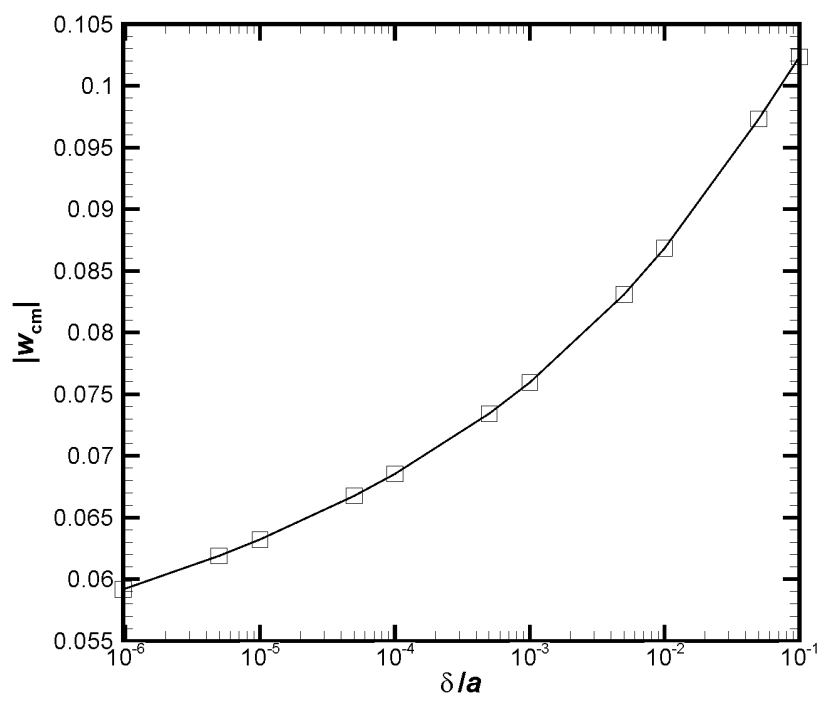

Figure 2: The average migration velocity, $w_{c m}$, of the particle pair as a function of inter-particle separation for the case where the angle between the lineof-centers and the ambient flow direction is $45^{\circ}$.

with the ambient flow direction. Similar plots can be constructed at different angles so that, at any given angle, the particle pair is always migrating faster towards the high shear rate region upon separation than towards the low shear rate region upon approach. This seems to contradict the end result that the net particle migration is towards the low shear rate region of the flow field. The resolution of this apparent contradiction lies in the fact that the time of approach is much longer than the time of separation. The reason for this is that the particles approach each other and, when the roughness limit is reached, the particles begin to roll over each other separated by the roughness distance until their line of centers is vertically aligned. At that point, the particles will separate in the horizontal direction as they are no longer constrained by the separation distance. Ancillary to the particles no longer rolling over each other is that the separation velocity in the horizontal direction is significantly enhanced. For example, for the case considered in Fig. 1 with roughness $\varepsilon / a=0.01$, the time of approach is 62.6 seconds whereas the time of separation is 10.4 seconds. That is, the particles spend far more time migrating towards the low shear rate region (albeit at a slower migration velocity) than they spend migrating towards the high shear rate region.

Another way to look at the interaction between the two spheres in Poiseuille flow is to plot the vertical location of the center of mass of the particle pair, $z_{c m}$, as a function of the absolute value of horizontal center-to-center separation, $|\Delta x|$, as shown in Fig. 3. As seen in the inset of the figure, there is a range of values of $|\Delta x|$ for which the particle pair is actually closer to the high shear rate region of the flow field on separation compared to approach. This is caused by 
the particles being slightly farther apart on separation compared to approach and, for a given $|\Delta x|$, migrating slightly faster towards the high shear rate region on separation compared to approach. This offers a significant clue for the construction of particle trajectories in oscillating Poiseuille flow with short periods such that the particle pair actually migrates towards the high shear rate region of the flow field as described below.

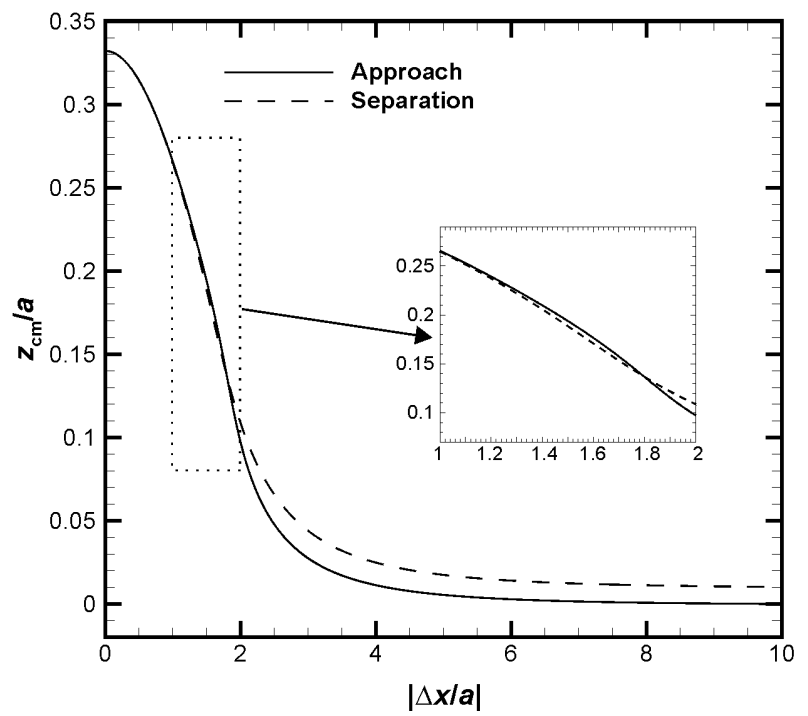

Figure 3: The vertical location of the center of mass, $z_{c m} / a$, as a function of $|\Delta x / a|$ for two spheres suspended in nonlinear shear flow with initial positions $(-5.0,0.0,0.05)$ and $(5.0,0.0,-0.05)$. The inset shows details of the graph for $1<|\Delta x / a|<2$.

We consider particles starting at various positions associated with points on the left side of the curve $(\Delta x / a<0)$ in Fig. 1 and then run the simulation in the forward direction for twice the time it takes the particle pair to travel from their initial position to $\Delta x / a=0$. The flow is then reversed for the same amount of time and the net distance that the center of mass of the particle pair travels, $\Delta z_{c m}$, is recorded. For the ambient flow field used previously, $|u(z)|=$ $1.08 \overline{3}-0.75 *(z-0.6 \overline{6})^{2}$, the net permanent vertical migration, $\Delta z_{c m}$, is plotted against the period of oscillation $T$ in Fig. 4. As seen in the figure, the net permanent migration changes sign at a critical period, $T_{c}$ of approximately $T_{c}=22$ seconds. For periods of oscillation less than $T_{c}$, the net migration of the particle pair is towards the high shear rate region of the flow field whereas, for periods of oscillation greater than $T_{c}$, the net migration of the particle pair is towards the low shear rate region of the flow field. In particular, we have removed the discrepancy between the approach and separation time. 


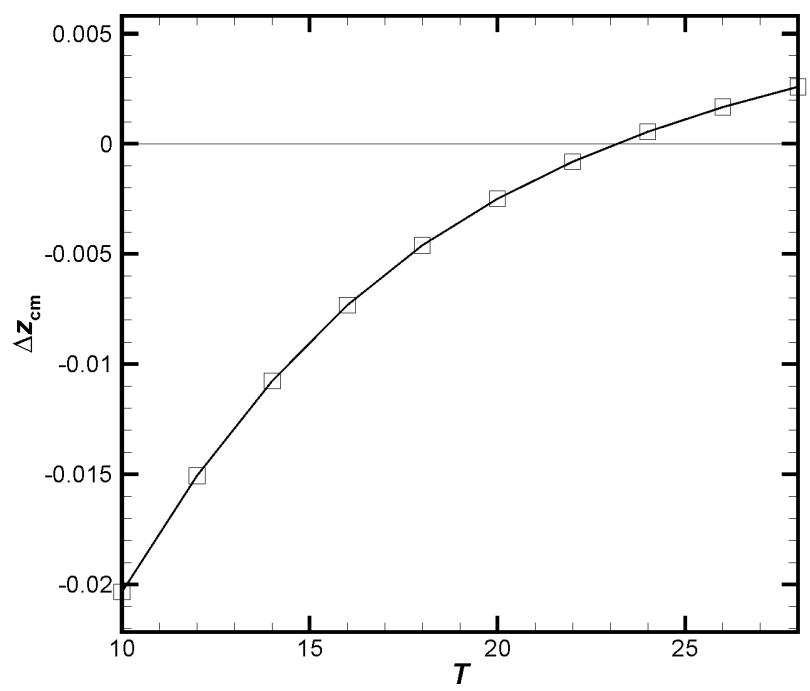

Figure 4: The net permanent vertical migration, $\Delta z_{c m}$, as a function of oscillation period, $T$.

This phenomenon of particles migrating towards the high shear rate region of the flow field observed in Poiseuille flow has not been observed in oscillating Couette flow, even though particle motion in oscillating Couette flow has been extensively studied [10]. A conjecture as to why this phenomenon has been observed in oscillating Poiseuille flow but not in oscillating Couette flow is put forward here by considering a plot of particle separation versus average migration velocity similar to Fig. 2. Specifically, we consider an ambient Couette velocity field $u_{\theta}=-r / 3+1323 / r$ and an ambient Poiseuille velocity field again given by $u(z)=1.08 \overline{3}-0.75 *(z-0.6 \overline{6})^{2}$. The Couette velocity field is actually taken from previous physical experiments [4]. The angle that the line-of-centers of the particle pair and the ambient flow direction is $30^{\circ}$ in the case considered here. The average migration velocity, $w_{c m}$, is plotted as a function of the inter-particle separation, $\delta$, in Fig. 5.

Although the two curves in the figure are qualitatively the same, there are a couple of interesting differences. For both the Poiseuille flow case and Couette flow case, the migration velocity increases with increasing separation to a point, reaches a maximum, and then decreases. It is this increase in migration velocity with increasing separation that can result in, as discussed above, a net migration towards the high shear rate region of the flow field for small period oscillatory flows. However, the rate of increase with increasing separation is much smaller for Couette flow compared to Poiseuille flow. Further, the maximum value of the interparticle separation $\delta / a$ for which $w_{c m}$ is increasing is much smaller for Couette flow. In particular, this maximum value occurs at $\delta / a \approx 0.005$ for Couette flow. 


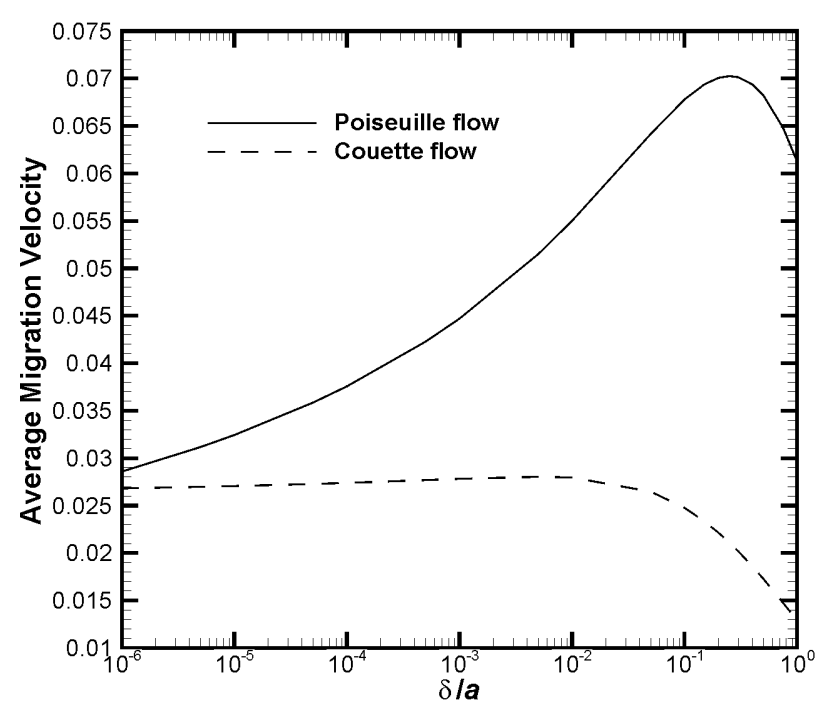

Figure 5: The average migration velocity of the particle pair as a function of interparticle separation for the case where the angle of the particle pair lineof-centers with the ambient flow is $30^{\circ}$.

If the roughness of the particle is of the same order of magnitude, the reversal of migration direction will not be experienced regardless of oscillation period.

\section{Conclusion}

The migration of a pair of spheres in oscillatory Poiseuille and Couette flow has been carefully analyzed using a high fidelity semi-analytical solution of two spheres in arbitrary shear flow. It has been demonstrated that the spheres migrate towards the low shear rate region of the flow field on approach and towards the high shear rate region of the flow field on separation. Particle roughness causes an asymmetry in the trajectory of the center of mass of the particle pair, which results in a net migration. In general, roughness causes the two spheres to be slightly farther apart on separation compared to approach. There are two competing factors for the net migration of the particle pair. The first factor is that the migration rate at a given angle between the line-of-centers of the particle pair and the ambient flow increases with increasing separation for moderate separations. This factor contributes to the net migration being towards the high shear rate region of the flow field. The second factor is that the separation time to cover a specified $|\Delta x|$ is generally much shorter than the approach time for the same $|\Delta x|$. For non-oscillating flows, this second factor contributes to the net migration being towards the low shear rate region of the flow field. For oscillating flows with large period, the second factor is predominant whereas, for oscillating flows with short period, the first factor is predominant. This analysis then presents a first-principle 
explanation for the observed change in migration direction for relatively dense suspensions. It was also shown that, although it would be theoretically possible to observe a reversal in migration direction for oscillatory Couette flow, it would be difficult to observe in practice for two reasons. First, the increase in migration velocity with increasing separation is very slight for Couette flow and, second, the maximum separation for which this increase is observed may never be experienced because of particle roughness.

\section{References}

[1] Phillips, R. J., Armstrong, R. C., Brown, R. A., Graham, A. L., and Abbott, J. R., "A constitutive equation for concentrated suspension that accounts for shear-induced particle migration," Phys. Fluids A, 4 (1), 34-40, 1992.

[2] Subia, S. R., Ingber, M. S., Mondy, L. A., Altobelli, S. A., and Graham, A. L., "Modelling of concentrated suspensions using a continuum constitutive equation," J. Fluid Mech, 373, 193-219, 1998.

[3] Ingber, M. S., Graham, A. L., Mondy, L. A., and Fang, Z., "An improved constitutive model for concentrated suspensions accounting for shearinduced particle migration rate dependence on particle radius," Int. J. Multi. Flow, 35(3), 270-276, 2009.

[4] Tetlow, N., Graham, A. L., Ingber, M. S., Subia, S. R., Mondy, L. A., and Altobelli, S. A., "Particle migration in a Couette apparatus: Experiment and modeling," J. Rheol., 42(2), 307-327, 1998.

[5] Butler, J. E., Majors, P. D. and Bonnecaze, R. T., "Observations of shearinduced particle migration for oscillatory flow of a suspension within a tube," Phys. Fluids, 11(10), 2865-2877, 1999.

[6] Morris, J. F., “Anomalous migration in simulated oscillatory pressure-driven flow of a concentrated suspension," Phys. Fluids, 13(9), 2457-2462, 2001.

[7] Ingber, M. S. and Zinchenko, A., "Semi-analytic solution of the motion of two spheres in arbitrary shear flow," Int. J. Multi. Flow, 42, 152-163, 2012.

[8] Ingber, M. S., Feng, S., Graham, A. L. and Brenner, H., "The analysis of self-diffusion and migration of rough spheres in nonlinear shear flow using a traction-corrected boundary element method," J. Fluid Mech., 598, 267-292, 2008.

[9] DaCunha, F. R. and Hinch, E. J., "Shear-induced dispersion in a dilute suspension of rough spheres," J. Fluid Mech., 309, 211-223, 1996.

[10] Pine, D. J., Gollub, J. P., Brady, J. F. and Leshansky, A. M., "Chaos and threshold for irreversibility in shear suspensions," Nature, 438, 997-1000, 2005. 